\title{
Real-time intraoperative detection of melanoma lymph node metastases using recombinant vaccinia virus GLV-1h68 in an immunocompetent animal model
}

\author{
Kaitlyn J. Kelly ${ }^{1}$, Peter Brader ${ }^{2}$, Yanghee Woo ${ }^{1}$, Sen $\mathrm{Li}^{1}$, Nanhai Chen ${ }^{3}$, Yong A. Yu ${ }^{3}$, \\ Aladar A. Szalay ${ }^{3,4,5}$, and Yuman Fong ${ }^{1}$ \\ ${ }^{1}$ Department of Surgery, Memorial Sloan-Kettering Cancer Center, New York, NY \\ 2 Department of Radiology, Memorial Sloan-Kettering Cancer Center, New York, NY \\ ${ }^{3}$ Genelux Corporation, San Diego Science Center, San Diego, CA \\ ${ }^{4}$ Virchow Center for Experimental Biomedicine, University of Wuerzburg, Wuerzburg, Germany \\ ${ }^{5}$ Institute for Biochemistry and Institute for Molecular Infection Biology, University of Wuerzburg, \\ Wuerzburg, Germany
}

\begin{abstract}
There is a clinical need for improved intraoperative detection of lymph node metastases from malignant melanoma (MM). We aimed to investigate the use of recombinant vaccinia virus GLV-1h68, expressing green fluorescent protein (GFP), for real-time intraoperative detection of melanoma lymph node metastases in an immunocompetent animal model. Mice bearing foot pad tumors received intratumoral injections of GLV-1h68 and 48 hours later were evaluated for popliteal lymph node metastasis using noninvasive bioluminescence imaging and fluorescence imaging. Histologic analysis of lymph nodes was performed to determine sensitivity and specificity of virus-mediated detection. Intratumoral injection of GLV-1h68 into primary foot pad melanoma tumors resulted in viral transmission to popliteal lymph nodes, infection of lymphatic metastases, and transgene expression that was reliably and easily detected. Histologic confirmation demonstrated favorable operating characteristics of this assay (sensitivity $80 \%$, specificity $100 \%$, positive predictive value [PPV] $100 \%$, negative predictive value [NPV] $91 \%$ ). Detection of marker gene expression by GLV-1h68 allowed the detection of lymphatic metastases in an immunocompetent animal model of MM. This assay is rapid, sensitive, specific, and easy to perform and interpret. As a candidate gene therapy virus for killing cancer, GLV-1h68 may also have significant concomitant diagnostic utility in the staging of cancer patients.
\end{abstract}

\section{Keywords}

Malignant melanoma; sentinel lymph node biopsy; oncolytic virus

\footnotetext{
Correspondence should be addressed to: Yuman Fong, Department of Surgery, Memorial Sloan-Kettering Cancer Center, 1275 York Avenue, H-1207, New York, NY 10065, (212) 639-7119, FAX (212) 794-5852, fongy@mskcc.org and Aladar A. Szalay, Genelux Corporation, San Diego Science Center, 3030 Bunker Hill Street, Suite 310, San Diego, CA 92109, (858) 483 0024, x203, FAX (858) 483-0026.

This manuscript describes the first effective use of transgene expression by an oncolytic vaccinia virus for detection and staging of cancer in an immunocompetent animal model.
} 


\section{Introduction}

Malignant melanoma is the most lethal form of skin cancer, accounting for $75 \%$ of skin cancer deaths in 2007 (1). At present, approximately 60,000 new cases of melanoma are diagnosed yearly. Early diagnosis and treatment are paramount. The 5-year survival rate for patients with localized, early stage disease is $99 \%$, but this drops to $65 \%$ and $15 \%$ for patients with regional and distant stage disease, respectively.

The most important predictor of survival in patients is the tumor status of the regional lymph node basin (2;3). Sentinel lymph node biopsy (SLNB) is the standard procedure for determining regional lymph node status for patients with intermediate-thickness melanoma. SLNB is a procedure whereby dye or radioactive tracer is injected into the site of cancer. As the dye travels along the lymphatics, the first regional lymph node that shows dye uptake or radioactive tracer uptake is biopsied. This test that maps lymphatic trafficking is used to identify the node with the highest likelihood of cancer for biopsy and histologic documentation of presence or absence of nodal metastases. Detection of subclinical lymph node metastases identifies those patients in need of complete lymphadenectomy, the only therapeutic option for local control.

SLNB, however, has limitations. Intraoperative analysis of sentinel lymph nodes (SLNs) by frozen section lacks sensitivity and frequently fails to identify nodal disease (4-6). The majority of patients must undergo lymphadenectomy as a second procedure, after detection of nodal disease by permanent section, immunohistochemistry, and/or molecular analysis.

Oncolytic vaccinia viruses are replication-competent, attenuated viruses that selectively infect and kill cancer cells, sparing normal cells. The therapeutic efficacy of these viruses has been demonstrated in a wide variety of human malignancies in vitro and in experimental models (7-13). Vaccinia viruses are well-suited to act as oncolytic agents as they have a natural tropism for targeting tumors when administered in vivo. In addition, the large genome of vaccinia virus can accommodate the insertion of multiple transgenes without loss of stability or diminished replicative ability. GLV-1h68 is an oncolytic vaccinia virus genetically modified by insertion of various marker genes, one of which replaces the gene encoding viral thymidine kinase (TK). As a result of these modifications, this virus can only productively infect and replicate in rapidly dividing, transformed cells with upregulated cellular TK. GLV-1h68 carries the marker genes encoding for GFP, Ruc, and $\beta$-gal. Expression of these transgenes by cancer cells infected with the virus can be detected by fluorescence and bioluminescence imaging modalities.

This study investigates the potential of GLV-1h68 to track and detect lymph node metastases after direct injection into primary melanoma tumors, and to enable real-time intraoperative detection of lymph node metastases by fluorescence imaging for GFP expression in an immunocompetent animal model.

\section{Materials and Methods}

\section{Cell lines}

The human metastatic melanoma cell lines SK-Mel 2, SK-Mel 119, and SK-Mel 197 were a generous gift from Dr. Alan Houghton at Memorial Sloan-Kettering Cancer Center (MSKCC). They were grown in Roswell Park Memorial Institute (RPMI) medium supplemented with $10 \%$ fetal calf serum (FCS), $100 \mathrm{U} / \mathrm{mL}$ penicillin, and $100 \mathrm{mg} / \mathrm{mL}$ streptomycin. The syngeneic malignant melanoma cell line B16-F10 was obtained from the American Type Culture Collection (ATCC, Rockville, MD) and grown in Dulbecco's modified Eagle's medium high glucose (DME-HG) supplemented with 10\% FCS, 100 U/ 
$\mathrm{mL}$ penicillin, $100 \mathrm{mg} / \mathrm{mL}$ streptomycin, and $1.5 \mathrm{~g} / \mathrm{L}$ sodium bicarbonate. All cells were maintained in a humidified incubator at $37{ }^{\circ} \mathrm{C}$ supplied with $5 \% \mathrm{CO}_{2}$.

\section{Virus strain}

GLV-1h68 is a replication-competent, recombinant vaccinia virus derived from the vaccinia virus LIVP strain (Lister strain from the Institute of Viral Preparations, Moscow), whose construction was previously described (13). GLV-1h68 carries 3 gene cassettes inserted into the viral genome: a Renilla luciferase-GFP ( $r u c-g f p$ ) fusion cassette in the F14.5LF14.5L locus, a reverse inserted human transferrin receptor and $\beta$-galactosidase cassette in the $J 2 R$ locus (which encodes TK), and a $\beta$-glucuronidase cassette in the A56R locus (encoding hemagglutinin).

\section{GFP expression}

Cells were plated at $2 \times 10^{4}$ per well in 12-well plates in $1 \mathrm{~mL}$ of medium per well. After 6 hours of incubation, GLV-1h68 in $100 \mu \mathrm{L}$ Dulbecco's modified Eagle's medium (DMEM) with $2 \%$ FCS per well was added at multiplicities of infection (MOIs) of 10, 1.0, and 0.1 for B16-F10 cells, and 1.0, 0.1, and 0.01 for human cell lines. All conditions were performed in triplicate. At time intervals of 6,12, 24, and 48 hours postinfection, cells were examined with a fluorescence inverted microscope (Nikon Eclipse TE300, Nikon Japan) for GFP expression and photographs taken.

\section{5-Bromo-4-chloro-3-indolyl-B-D-galactopyranoside (X-gal) cytochemistry}

Cells were plated at $2 \times 10^{4}$ per well in 24-well plates with $1 \mathrm{~mL}$ of medium per well. After incubation for 6 hours, cells were infected with GLV-1h68 at an MOI of 5. At varying intervals, cells were fixed with $1 \%$ glutaraldehyde for 5 minutes. Cells were stained with 1 $\mathrm{mg} / \mathrm{mL} \mathrm{X-gal} \mathrm{in} \mathrm{an} \mathrm{iron} \mathrm{solution} \mathrm{of} 5 \mathrm{mmol} / \mathrm{L} \mathrm{K} \mathrm{Fe}_{4}(\mathrm{CN})_{6}, 5 \mathrm{mmol} / \mathrm{L} \mathrm{K}_{3} \mathrm{Fe}(\mathrm{CN})_{6}$, and 2 $\mathrm{mmol} / \mathrm{L} \mathrm{MgCL}_{2}$ for 4 hours. Cells were then washed with phosphate-buffered saline (PBS) and examined by light microscopy.

\section{$\beta$-Galactosidase assays}

Cells were plated at $1 \times 10^{4}$ per well in 96-well plates with $100 \mu \mathrm{L}$ of medium per well. After incubation for 6 hours, human cell lines were infected with GLV-1h68 at MOI 1.0, and B16-F10 at MOI 10.0 in $50 \mu \mathrm{L}$ of medium per well. At varying intervals, cells were lysed and $\beta$-galactosidase activity measured with an Enhanced $\beta$-Galactosidase assay kit (Gene Therapy Systems, San Diego, CA) using a spectrophotometer (EL321e, Bio-Tek Instruments, Winooski, VT) at $570 \mathrm{~nm}$. All samples were measured in triplicate.

\section{Luciferase assays}

Cells were plated at $2 \times 10^{4}$ per well in 96-well plates in $100 \mu \mathrm{L}$ of medium per well. After incubation for 6 hours, cells were infected with GLV-1h68 at an MOI of 1.0 (human cell lines) or 10.0 (syngeneic cell line) for varying intervals. Coelenterazine $(0.25 \mu \mathrm{g}$; Biotium, Hayward, CA) diluted in $50 \mu \mathrm{L}$ of PBS was added per well for 10 minutes and luciferase activity was detected with a cooled high-sensitivity charge coupled device (CCD) camera (Xenogen IVIS 100 Caliper, Hopkinton, MA). Emitted photons were measured for 30 seconds from ventral views. Images were analyzed with Living Image 2.5 software (Caliper, Hopkinton, MA). All samples were analyzed in triplicate.

\section{Cytotoxicity assays}

Cells were plated at $2 \times 10^{4}$ cells per well in 12-well plates with $1 \mathrm{~mL}$ of medium. After incubation for 6 hours, cells were infected with GLV-1h68 at MOIs of 0, 0.01, 0.1, and 1.0 for the human melanoma cell lines and $0,1,10,25,50$, and 100 for the syngeneic cell line. 
Vehicle-treated cells served as controls. Viral cytotoxicity was tested at daily intervals. Medium was removed daily and cells were lysed with $1.35 \%$ Triton-X solution to release intracellular lactate dehydrogenase (LDH). Standard LDH assay was performed using the Cytotox 96 nonradioactive cytotoxicity assay (Promega, Madison, WI) and a microplate reader (EL321e, Bio-Tek Instruments). Results are expressed as the percentage of surviving cells relative to controls wells.

\section{Establishment of an immunocompetent animal model of melanoma lymphatic metastasis}

Four-to-6-week old homozygous C57BL/6J-Tyr $\mathrm{C}-2 \mathrm{~J} / \mathrm{J}$ albino mice (carrying a spontaneous mutation in the tyrosinase gene) were purchased from Jackson Laboratories (Bar Harbor, ME). All animals were provided with food and water ad libitum. Animals received humane care in accordance with the "Guide for the care and use of laboratory animals (NIH)" and the animal protocol was approved by the animal care and use committee (IACUC) at MSKCC. Anesthesia was induced by a mixture of Isoflurane $(2 \mathrm{~L} / \mathrm{min})$ and oxygen $(4 \mathrm{~L} /$ $\mathrm{min})$ in an induction chamber and was maintained by a nasal cone. B16-F10 cells $\left(2 \times 10^{5}\right.$ in $50 \mu \mathrm{L}$ PBS) were injected to the right hind footpad. Gentle pressure was applied to the right hind limb just above the foot during injection to prevent extravasation of the cell suspension out of the footpad and up the leg.

\section{Lymph node imaging}

Detection of Fluorescence-Three weeks after tumor implantation, 20 animals with tumor and 5 control animals (previously injected with PBS only) received intratumoral injections of $1 \times 10^{7}$ pfu of GLV-1h68 in $50 \mu \mathrm{L}$ of PBS. Forty-eight hours after injection of virus, animals were sacrificed with $\mathrm{CO}_{2}$ inhalation. The medial aspect of the right hind limb was surgically exposed from the site of the footpad tumor to the right groin. Animals were imaged using the Leica MZFL3 Stereomicroscope (Leica Microsystems, Wetzlar, Germany) in both bright-field and fluorescent modes. The Retiga EX digital CCD camera (Qimaging, Burnaby, Canada) was used for image capture.

Detection of Bioluminescence-Just prior to sacrifice for fluorescence imaging, animals were anesthetized with a mixture of Isoflurane $(2 \mathrm{~L} / \mathrm{min})$ and oxygen $(4 \mathrm{~L} / \mathrm{min})$ in an induction chamber. Anesthesia was maintained by a nasal cone. Animals underwent retro-orbital injection of coelenterazine ( $5 \mu \mathrm{L}$ of $0.5 \mu \mathrm{g} / \mu \mathrm{L}$ solution diluted in $95 \mu \mathrm{L}$ PBS). Anesthetized animals were then placed in a dark box for photon counting (IVIS 100 Caliper, Hopkinton, MA). Emitted photons were measured for 30 seconds. Images were analyzed with Living Image 2.5 software (Caliper, Hopkinton, MA). Animals harboring tumor treated with intratumoral injection of PBS only $(\mathrm{n}=3)$ and animals without tumor treated with GLV-1h68 $(\mathrm{n}=3)$ served as controls.

\section{Histologic Analysis of Lymph Nodes}

Immediately after imaging, lymph nodes were harvested and frozen in Tissue-Tek Optimal Cutting Temperature (O.C.T.) Compound (Sakura Finetek USA, Inc., Torrance, CA). Tissues were cut into 5 - $\mu \mathrm{m}$-thick sections on glass slides. Cryosections were fixed and stained with hematoxylin and eosin $(\mathrm{H} \& \mathrm{E})$ and $\mathrm{X}$-gal $(1 \mathrm{mg} / \mathrm{mL})$ in an iron solution of 5 $\mathrm{mmol} / \mathrm{L} \mathrm{K}{ }_{4} \mathrm{Fe}(\mathrm{CN})_{6}, 5 \mathrm{mmol} / \mathrm{L} \mathrm{K}_{3} \mathrm{Fe}(\mathrm{CN})_{6}$, and $2 \mathrm{mmol} / \mathrm{L} \mathrm{MgCL}_{2}$ to identify virally mediated $l a c z$ expression. Cryosections from each sample were also fixed and left unstained for light microscopic examination for the presence of black-pigmented melanoma cells (14). 


\section{Results}

\section{GLV-1h68 infection allows successful transgene expression in B16-F10 melanoma cells and in a panel of human melanoma cell lines}

Fluorescence Microscopy-In all cell lines, GFP expression increased with increasing MOI. At all MOIs tested, the percentage of GFP-expressing cells increased gradually over time (Fig. 1). Very few cells expressed GFP at 6 hours after infection, but by 12 hours GFP expression was visible in all cell lines. Of the human melanoma cell lines, SK-Mel 119 demonstrated the greatest GFP expression, followed by SK-Mel 2 and SK-Mel 197. B16F10 cells showed very few GFP-expressing cells at MOI 1.0, but significant expression at all time points at MOI 10. In all cell lines tested, abundant GFP expression was present by 48 hours after virus infection.

Luciferase Assay-Luciferase activity paralleled GFP and $\beta$-galactosidase expression, gradually increasing over time. SK-Mel 119 cells demonstrated the greatest luciferase expression with $3.6 \times 10^{7} \pm 5 \times 10^{6}$ photons $/ \mathrm{s} / \mathrm{cm}^{2} / \mathrm{sr}$ at 48 hours after infection with MOI 1.0. B16-F10 cells displayed $2.4 \times 10^{7} \pm 9.2 \times 10^{6} \mathrm{photons} / \mathrm{s} / \mathrm{cm}^{2} / \mathrm{sr}$ at 48 hours after infection with MOI 10.0 (Fig. 2). Quantified luciferase activity correlated well with visual findings by GFP microscopy. The genes encoding Ruc $(r u c)$ and GFP $(g f p)$ are present in the same gene fusion cassette in the GLV-1h68 virus genome and so are expected to display similar levels of expression.

$X$-Gal Cytochemistry and $\beta$-Galactosidase Assay-X-gal staining demonstrated results similar to GFP fluorescence microscopy. For all MOIs tested, the percentage of blue cells increased gradually over time, with very few blue cells at 6 hours, more at 12 hours, and an abundance of cells by 48 hours in all cell lines. In addition to this qualitative data, $l a c z$ expression was assessed quantitatively with a $\beta$-galactosidase activity assay. At 12 hours, low levels of $\beta$-galactosidase were measured $(0.030-0.080 \mathrm{mU})$. Increasing levels were measured at 24 and 48 hours. At 48 hours after infection with MOI 1.0, SK-Mel 2 demonstrated the greatest amount of $\beta$-galactosidase $(2.03 \pm 0.15 \mathrm{mU})$, followed by SK-Mel $119(1.69 \pm 0.24 \mathrm{mU})$, and SK-Mel $197(0.39 \pm 0.09 \mathrm{mU})$. At 48 hours after infection with MOI 10.0, B16-F10 cells demonstrated $1.64 \pm 0.15 \mathrm{mU} \beta$-galactosidase activity. As expected, determination of $\beta$-galactosidase enzyme activity correlated with qualitative visual findings by X-gal cytochemistry (Fig. 3).

\section{GLV-1h68 is cytotoxic to human and murine melanoma cells}

All human melanoma cell lines demonstrated significant sensitivity to GLV-1h68, with $>90 \%$ cells killed in SK-Mel 119 and SK-Mel 197 cell lines and 85\% in SK-Mel 2 by day 7 at MOI 1.0. The murine melanoma cell line was significantly more resistant, requiring MOI 50 to achieve a successful cytotoxic effect. In all cell lines, the cytotoxic effect of the virus was dose dependent (Fig. 4).

\section{GLV-1h68-guided detection of lymph node metastases}

Fluorescence-Mediated Detection-We found that GLV-1h68-mediated GFP expression in primary melanoma tumors and lymph node metastases can be detected with fluorescence imaging in vivo. All 20 tumor-bearing mice demonstrated a strong fluorescent signal at the primary tumor site whereas control mice (those with PBS-treated tumors $(n=6)$ and those with no tumors that were virus treated; $n=6$ ) had no signals. Four of 20 mice (20\%) demonstrated a fluorescent signal at the right popliteal lymph node. Figure 5 illustrates these findings in animals with microscopic and gross nodal disease. No signals were detected over the thorax or other areas in any of the animals. 
Bioluminescence-Mediated Detection-We found that GLV-1h68-mediated luciferase expression in primary melanoma tumors and popliteal lymph nodes can be detected with bioluminescence imaging in vivo. Again, all 20 tumor-bearing, virus-treated animals demonstrated luminescence at the primary tumor site whereas control mice (those with PBS-treated tumors and those with no tumors but virus-treated; $n=6$ ) had no luminescent signals. The same 4 of 20 mice (20\%) demonstrated bioluminescence at the right popliteal area, indicating the presence of nodal metastasis (Fig. 6a).

\section{Histologic analysis of lymph nodes}

Histologic examination confirmed the presence of metastatic melanoma in popliteal lymph nodes in 5 of 20 (25\%) animals. Lymph node metastases were easily detected by light microscopic examination for the presence of black melanin pigmentation. Three of 5 animals had grossly detectable invasion of lymph nodes with nodal tissue nearly replaced by tumor. Two of the 5 had microscopic metastasis.

Four of these 5 animals (80\%) demonstrated measurable luminescent and fluorescent lymph node signals ( 2 with gross and 2 with microscopic nodal metastases). Areas of GFP expression localized to tumor foci in lymph nodes (Fig. 7). Absence of tumor and GFP in negative lymph nodes (as determined by fluorescent imaging) was similarly confirmed by microscopic analysis. Finally, X-gal cytochemistry detected GLV-1h68-mediated lacz expression in the popliteal lymph nodes of the same 4 animals with positive findings by bioluminescent and fluorescent imaging, further confirming successful transgene expression by the virus in areas of metastatic disease. The sensitivity, specificity, positive predictive value $[\mathrm{PPV}]$, and negative predictive value [NPV] of virally mediated fluorescent detection of nodal metastasis was $80 \%, 100 \%, 100 \%$, and $93 \%$, respectively (Table 1).

\section{Discussion}

Many oncolytic viruses have been designed for specific, targeted killing of cancer. The genetic alterations of many of these viruses include insertion of transgenes, such as immunestimulating genes for enhanced therapeutic efficacy or marker genes for monitoring of therapy. Vaccinia virus is particularly well suited for oncolytic therapy because it is known to be safe in humans as it has been given to millions of people with acceptable toxicity in the form of the smallpox vaccine, it has a large genome that is able to incorporate multiple transgenes, and it is highly immunogenic so that infected cancer cells will incite an immune response, further contributing to the anticancer effect $(15 ; 16)$. GLV-1h68 is an attenuated oncolytic vaccinia virus carrying 3 marker genes that has been shown to be highly cancer specific (13). We have demonstrated for the first time that GLV-1h68 successfully tracks lymph node metastases from malignant melanoma and allows for rapid and simple detection of metastatic disease in an immunocompetent animal model.

Multiple studies have shown that for patients with intermediate-thickness cutaneous melanoma, regional lymph node status is the most important predictor of recurrence and survival (17-20). The current standard technique of injecting dye or radiotracer for SLNB successfully identifies the sentinel node, but provides no information about the status of that node until resection and processing. It has been shown that intraoperative frozen section of SLNs of patients with cutaneous melanoma is only $38 \%$ sensitive and is therefore not justified for routine use (4-6). Imprint cytology has been investigated in attempts to improve sensitivity of intraoperative detection of sentinel node disease. Results have shown, however, that imprint cytology is equivalent to but not better than frozen section analysis, with reported sensitivities of 38\% (21) and 33\% (22) for detection of melanoma nodal metastases. Without a reliable assay for intraoperative assessment, melanoma patients with positive SLNs must undergo lymph node dissection as a second procedure. Even permanent 
section histologic analysis has been shown to lack sensitivity relative to newer molecular detection techniques such as immunohistochemistry (IHC) and reverse-transcriptase polymerase chain reaction (RT-PCR) (23).

GLV-1h68 was able to track lymph node metastases 48 hours after injection into the primary tumor in an immunocompetent animal model of metastatic melanoma. Vaccinia virus mediated GFP expression offers the possibility of immediate identification of regional lymph nodes harboring metastatic disease in the operative setting by the presence or absence of easily identifiable green fluorescence. In our study, the transgenes ruc and lacz were used to confirm that green fluorescence detected in lymph nodes was due to GFP expression as opposed to autofluorescence.

We found that GLV-1h68-mediated, real-time fluorescence detection of nodal disease was $80 \%$ sensitive and $100 \%$ specific for identification of sentinel node disease. One animal with macroscopic disease of the popliteal lymph node did not display GLV-1h68 transgene expression. Of note, the fluorescent and bioluminescent signals from the primary footpad tumor in this animal were particularly weak when compared with those of other animals in the study, indicating that there may have been a technical problem with injection of virus in this animal.

It is also possible that GLV-1h68 may allow for intraoperative detection of nonsentinel node disease. Our model is limited for assessing this utility as the regional nodal basin draining the mouse footpad consists of a solitary popliteal node. The next lymph node in the drainage system is the inguinal node, which is significantly proximal. In our study, no animals had inguinal node metastases. An alternative model to test the ability of the virus to detect nonsentinel node disease would be a mammary fat pad or truncal tumor with multiple lymph nodes in a regional drainage basin. Eisenberg et al. demonstrated that GFP-expressing herpes simplex virus is able to identify nonsentinel node disease in a xenograft model of breast cancer metastatic to the axillary lymph nodes (24).

In addition to offering increased sensitivity of intraoperative detection, GLV-1h68 may confer an additional therapeutic advantage. A recombinant vaccinia virus, rV-B7.1, expressing a T-cell costimulatory molecule was shown to be cytotoxic to human melanoma cells and to produce biologic effects with an acceptable safety profile in a phase I clinical trial $(10 ; 25 ; 26)$. GLV-1h68 has been shown to be an effective oncolytic agent against human breast cancer, anaplastic thyroid cancer, and malignant pleural mesothelioma cell lines in vitro and in vivo $(11 ; 13)$. We have shown that GLV-1h68 was cytotoxic to malignant melanoma cell lines in vitro and in vivo (unpublished data).

Fluorescence detection of lymphatic metastases with oncolytic vaccinia virus is a novel, sensitive, and specific technique for identification of metastatic melanoma. Advantages of this method compared with standard techniques include real-time intraoperative detection and the potential for a simultaneous therapeutic effect. In addition, this assay is both easy to perform and interpret, and it is cost-effective. This technique may benefit an enormous population of melanoma patients at least by allowing SLNB and lymph node dissection to be performed in a single procedure, and potentially by offering enhanced staging accuracy and a therapeutic effect.

\section{Acknowledgments}

This research was supported grants R01 CA 76416 (Y.F) from the National Institute of Health, the Flight Attendant Medical Research Institute (F.A.M.R.I.), and by grant support to the Research and Development Division of Genelux Corporation (A.A.S). A financial conflict of interest does exist for co-authors affiliated with Genelux Corporation. Specifically, Dr. Yong A. Yu and Dr. Aladar Szalay are shareholders of and are employed by Genelux 
Corporation. Dr. Nanhai Chen is employed by Genelux Corporation. We would like to thank our editor, Meryl Greenblatt, for her outstanding assistance in this work.

\section{Abbreviations}

$\begin{array}{ll}\text { MM } & \text { Malignant melanoma } \\ \text { GFP } & \text { green fluorescent protein } \\ \text { SLNB } & \text { sentinel lymph node biopsy } \\ \text { PBS } & \text { phosphate buffered saline } \\ \text { LDH } & \text { lactate dehydrogenase } \\ \text { MOI } & \text { multiplicity of infection } \\ \text { PPV } & \text { positive predictive value } \\ \text { NPV } & \text { negative predictive value }\end{array}$

\section{Reference List}

1. Garcia, M.; Jemal, AWECMHYSRTMJ. Global Cancer Facts \& Figures 2007. Internet 2008(2007)Available from: URL: http://www.cancer.org/downloads/STT/Global_Cancer_Facts_and_Figures_2007_rev.pdf

2. Garrison M, Nathanson L. Prognosis and staging in melanoma. Semin Oncol. 1996 Dec; 23(6):72533. [PubMed: 8970594]

3. Amersi F, Morton DL. The role of sentinel lymph node biopsy in the management of melanoma. Adv Surg. 2007; 41:241-56. [PubMed: 17972569]

4. Koopal SA, Tiebosch AT, Albertus PD, Plukker JT, Schraffordt KH, Hoekstra HJ. Frozen section analysis of sentinel lymph nodes in melanoma patients. Cancer. 2000 Oct 15; 89(8):1720-5. [PubMed: 11042566]

5. Stojadinovic A, Allen PJ, Clary BM, Busam KJ, Coit DG. Value of frozen-section analysis of sentinel lymph nodes for primary cutaneous malignant melanoma. Ann Surg. 2002 Jan; 235(1):928. [PubMed: 11753047]

6. Andonian S, Meterissian SH, Watters AK, Loutfi A. A case of penile melanoma illustrating the low sensitivity of frozen sections in the assessment of sentinel lymph nodes. Can J Urol. 2003 Aug; 10(4):1947-9. [PubMed: 14503941]

7. McCart JA, Ward JM, Lee J, Hu Y, Alexander HR, Libutti SK, Moss B, Bartlett DL. Systemic cancer therapy with a tumor-selective vaccinia virus mutant lacking thymidine kinase and vaccinia growth factor genes. Cancer Res. 2001; 61(24):8751-7. [PubMed: 11751395]

8. Chalikonda S, Kivlen MH, O’Malley ME, Eric D, McCart JA, Gorry MC, Yin XY, Brown CK, Zeh HJ III, Guo ZS, Bartlett DL. Oncolytic virotherapy for ovarian carcinomatosis using a replicationselective vaccinia virus armed with a yeast cytosine deaminase gene. Cancer Gene Ther. 2008 Feb; 15(2):115-25. [PubMed: 18084242]

9. Guo ZS, Naik A, O’Malley ME, Popovic P, Demarco R, Hu Y, Yin X, Yang S, Zeh HJ, Moss B, Lotze MT, Bartlett DL. The enhanced tumor selectivity of an oncolytic vaccinia lacking the host range and antiapoptosis genes SPI-1 and SPI-2. Cancer Res. 2005; 65(21):9991-8. [PubMed: 16267024]

10. Kaufman HL, Deraffele G, Mitcham J, Moroziewicz D, Cohen SM, Hurst-Wicker KS, Cheung K, Lee DS, Divito J, Voulo M, Donovan J, Dolan K, et al. Targeting the local tumor microenvironment with vaccinia virus expressing B7.1 for the treatment of melanoma. Journal Of Clinical Investigation. 2005; 115(7):1903-12. [PubMed: 15937544]

11. Lin SF, Yu Z, Riedl C, Woo Y, Zhang Q, Yu YA, Timiryasova T, Chen N, Shah JP, Szalay AA, Fong Y, Wong RJ. Treatment of anaplastic thyroid carcinoma in vitro with a mutant vaccinia virus. Surgery. 2007 Dec; 142(6):976-83. [PubMed: 18063085] 
12. Yu YA, Shabahang S, Timiryasova TM, Zhang Q, Beltz R, Gentschev I, Goebel W, Szalay AA. Visualization of tumors and metastases in live animals with bacteria and vaccinia virus encoding light-emitting proteins. Nature Biotechnology. 2004; 22(3):313-20.

13. Zhang Q, Yu YA, Wang E, Chen N, Danner RL, Munson PJ, Marincola FM, Szalay AA. Eradication of solid human breast tumors in nude mice with an intravenously injected lightemitting oncolytic vaccinia virus. Cancer Res. 2007 Oct 15; 67(20):10038-46. [PubMed: 17942938]

14. Harrell MI, Iritani BM, Ruddell A. Tumor-induced sentinel lymph node lymphangiogenesis and increased lymph flow precede melanoma metastasis. Am J Pathol. 2007 Feb; 170(2):774-86. [PubMed: 17255343]

15. McFadden G. Poxvirus tropism. Nat Rev Microbiol. 2005; 3(3):201-13. [PubMed: 15738948]

16. Smith SA, Kotwal GJ. Immune response to poxvirus infections in various animals. Crit Rev Microbiol. 2002; 28(3):149-85. [PubMed: 12385498]

17. Zettersten E, Shaikh L, Ramirez R, Kashani-Sabet M. Prognostic factors in primary cutaneous melanoma. Surg Clin North Am. 2003 Feb; 83(1):61-75. [PubMed: 12691450]

18. Thompson JF, Shaw HM. The prognosis of patients with thick primary melanomas: is regional lymph node status relevant, and does removing positive regional nodes influence outcome? Ann Surg Oncol. 2002 Oct; 9(8):719-22. [PubMed: 12374653]

19. Francken AB, Accortt NA, Shaw HM, Wiener M, Soong SJ, Hoekstra HJ, Thompson JF. Prognosis and determinants of outcome following locoregional or distant recurrence in patients with cutaneous melanoma. Ann Surg Oncol. 2008 May; 15(5):1476-84. [PubMed: 18196345]

20. Gershenwald JE, Mansfield PF, Lee JE, Ross MI. Role for lymphatic mapping and sentinel lymph node biopsy in patients with thick ( $>$ or $=4 \mathrm{~mm}$ ) primary melanoma. Ann Surg Oncol. 2000 Mar; 7(2):160-5. [PubMed: 10761797]

21. Creager AJ, Shiver SA, Shen P, Geisinger KR, Levine EA. Intraoperative evaluation of sentinel lymph nodes for metastatic melanoma by imprint cytology. Cancer. 2002 Jun 1; 94(11):3016-22. [PubMed: 12115392]

22. Soo V, Shen P, Pichardo R, Azzazy H, Stewart JH, Geisinger KR, Levine EA. Intraoperative evaluation of sentinel lymph nodes for metastatic melanoma by imprint cytology. Ann Surg Oncol. 2007 May; 14(5):1612-7. [PubMed: 17287998]

23. Shivers S, Jakub J, Pendas S, Reintgen D. Molecular staging for patients with malignant melanoma. Expert Rev Anticancer Ther. 2007 Nov; 7(11):1665-74. [PubMed: 18020932]

24. Eisenberg DP, Adusumilli PS, Hendershott KJ, Chung S, Yu Z, Chan MK, Hezel M, Wong RJ, Fong Y. Real-time intraoperative detection of breast cancer axillary lymph node metastases using a green fluorescent protein-expressing herpes virus. Ann Surg. 2006 Jun; 243(6):824-30. [PubMed: 16772786]

25. Hodge JW, Abrams S, Schlom J, Kantor JA. Induction of antitumor immunity by recombinant vaccinia viruses expressing B7-1 or B7-2 costimulatory molecules. Cancer Res. 1994 Nov 1; 54(21):5552-5. [PubMed: 7522961]

26. Kaufman HL, Conkright W, Divito J Jr, Horig H, Kaleya R, Lee D, Mani S, Panicali D, Rajdev L, Ravikumar TS, Wise-Campbell S, Surhland MJ. A phase I trial of intra lesional RV-B7.1 vaccine in the treatment of malignant melanoma. Hum Gene Ther. 2000 May 1; 11(7):1065-82. [PubMed: 10811235] 


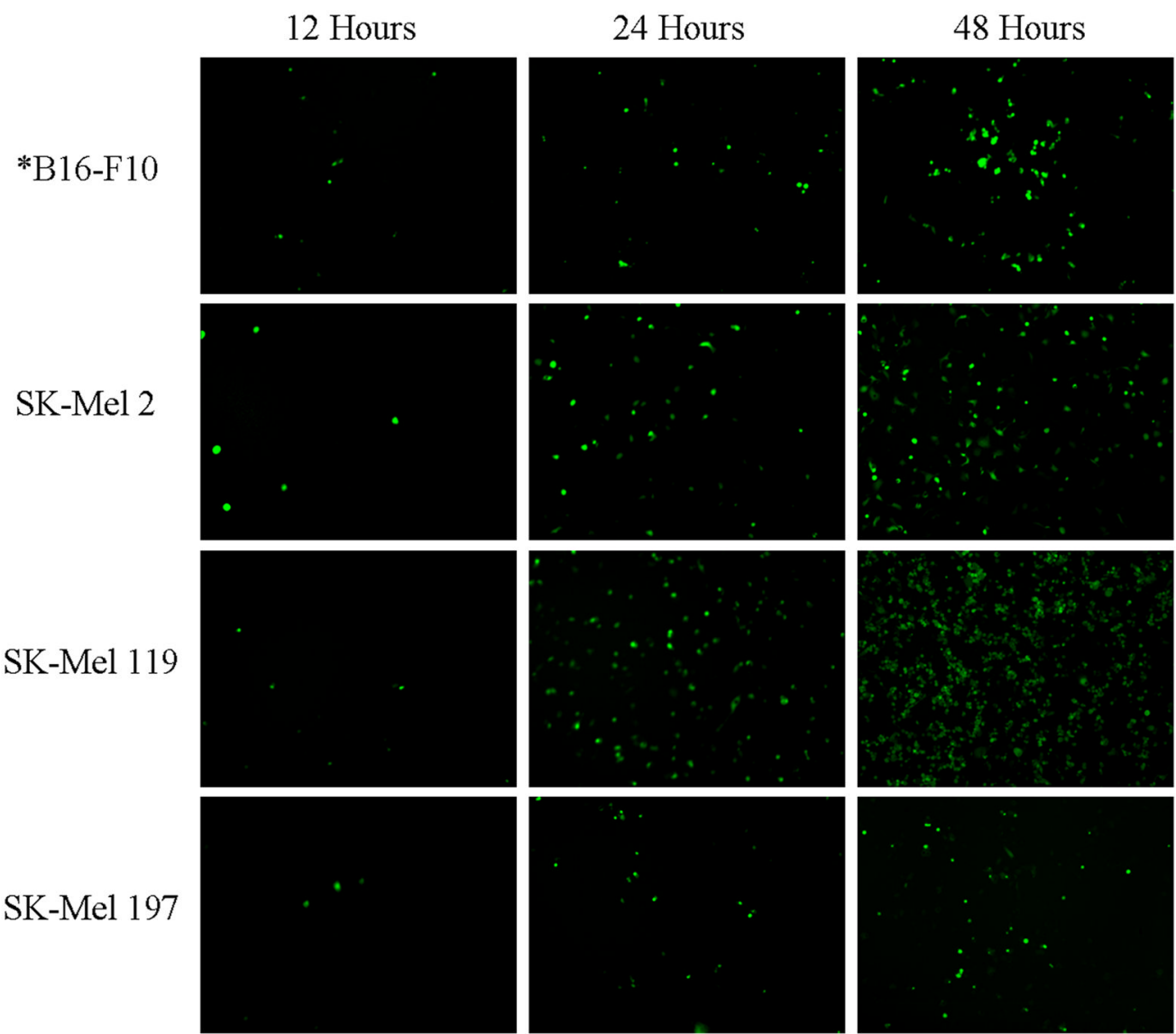

Figure 1. Fluorescence microscopy of malignant melanoma cell lines at various time points after infection with GLV-1h68

Few cells were GFP positive 12 hours after infection, but the percentage of positive cells increased over time with significant expression by 24 hours and abundant expression by 48 hours in all cell lines. GFP = green fluorescent protein. *B16-F10 murine melanoma cells were infected at MOI 10 whereas human cell lines were infected at MOI 1.0. 


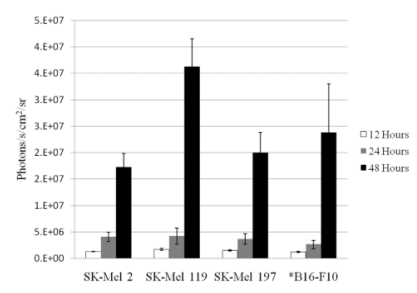

Figure 2. Quantitative luciferase assay in malignant melanoma cell lines at various time points after infection with GLV-1h68

All cell lines tested displayed increasing luciferase activity over time and all showed a large increase between 24 and 48 hours. Of the human cell lines, SK-Mel 119 displayed the greatest luciferase expression, followed by SK-Mel 2 and SK-Mel 197, which displayed similar amounts. B16-F10 murine melanoma cells displayed $2.38 \times 10^{7}$ photons $/ \mathrm{sec} / \mathrm{cm}^{2} / \mathrm{sr}$ 48 hours after infection. *B16-F10 murine melanoma cells were infected at MOI 10 whereas human cell lines were infected at MOI 1.0. 


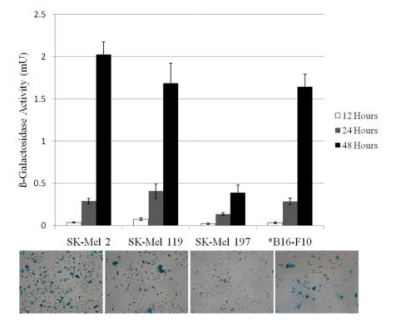

Figure 3. Quantitative $\beta$-galactosidase activity assay in malignant melanoma cell lines at various time points after infection with GLV-1h68

In all cell lines tested, $\beta$-galactosidase activity increased over time after infection with GLV-1h68. Of the human cell lines, SK-Mel 2 and SK-Mel 119 demonstrated the greatest activity at 48 hours after infection, and SK-Mel 197 displayed the least. B16-F10 murine melanoma cells demonstrated $1.64 \pm 0.15 \mathrm{mU} \beta$-galactosidase activity at 48 hours. X-gal staining demonstrated similar qualitative results as illustrated by the images of each cell line stained 48 hours after infection with virus. *B16-F10 murine melanoma cells were infected at MOI 10 whereas human cell lines were infected at MOI 1.0. 


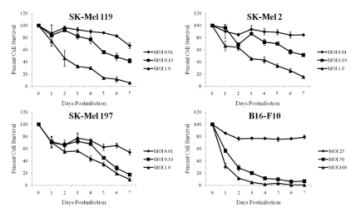

Figure 4. Cytotoxicity of human and murine melanoma cell lines to GLV-1h68 in vitro All 3 human melanoma cell lines demonstrated near complete cytotoxicity $(85 \% 95 \%$ cell kill) by day 7 at MOI 1.0. B16-F10 cells required higher doses of virus to achieve a cytotoxic effect, with $80 \%$ and $90 \%$ cell kill achieved by days 3 and 4, respectively, at MOI 50. 


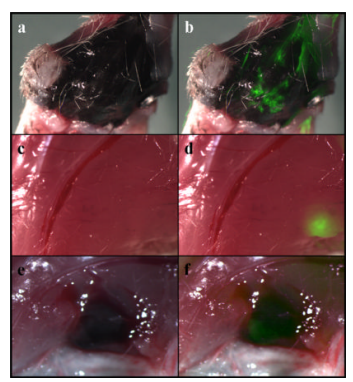

Figure 5. Detection of sentinel lymph node metastases with GLV-1h68-mediated fluorescence ( $a$ and b) Bright-field and overlay fluorescent images, respectively, of a primary footpad melanoma tumor. (c) Bright-field image of the right popliteal area of an animal with microscopic lymph node metastasis. (d) Corresponding overlay fluorescent image showing bright green fluorescence at the popliteal lymph node. (e) Bright-field image of popliteal lymph node with macroscopic melanoma metastasis. (f) Corresponding overlay fluorescent image showing virally mediated GFP expression at the involved lymph node. 


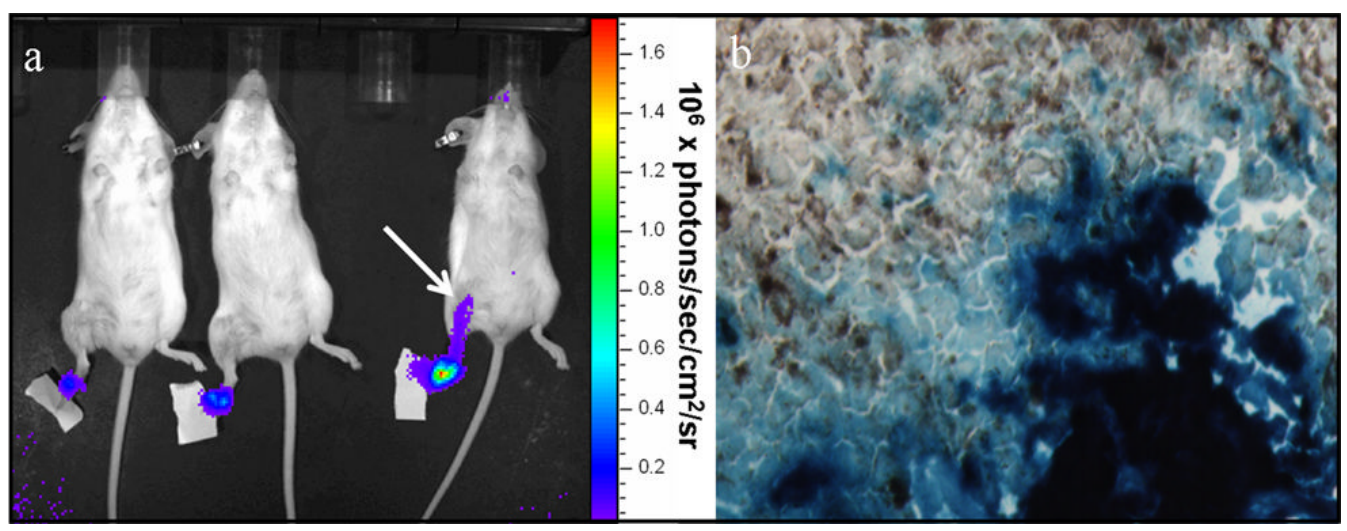

Figure 6. Detection of sentinel lymph node metastasis with alternative GLV-1h68-mediated Ruc and $\beta$-gal activities

(a) Bioluminescence images of 3 animals with primary footpad melanoma tumors 48 hours after intratumoral injection with GLV-1h68. The 2 animals on the left had disease limited to the footpad whereas the animal on the right had popliteal lymph node metastasis (arrow). (b) Positive X-gal staining in a popliteal lymph node harboring metastatic melanoma. 


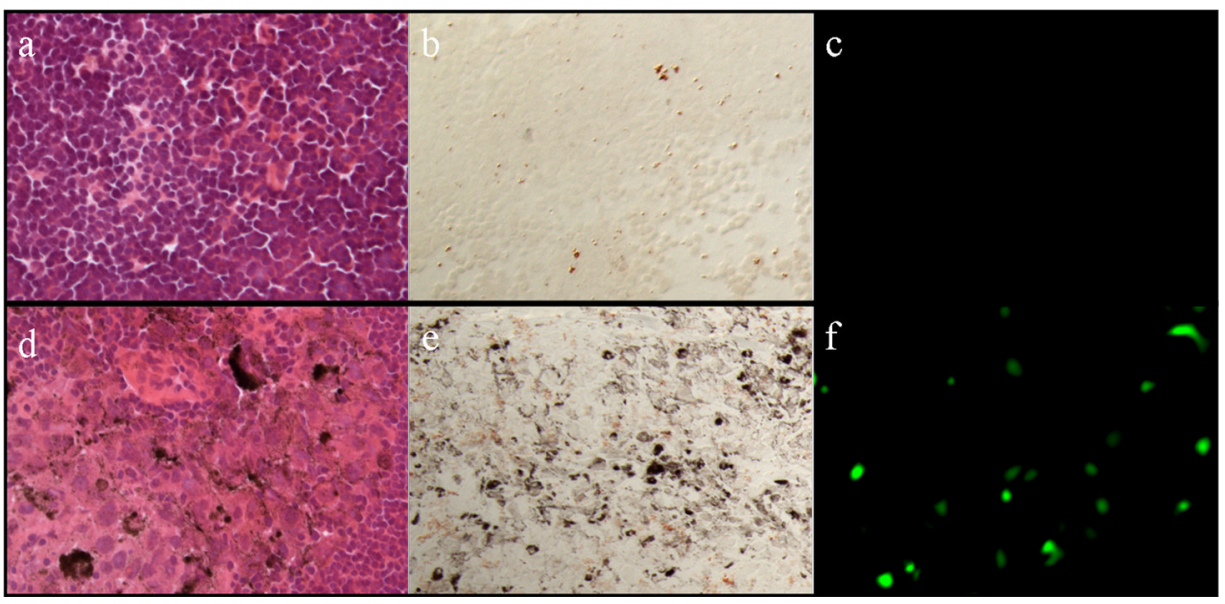

Figure 7. Histologic analysis of sentinel lymph nodes by hematoxylin and eosin (H\&E) staining, fluorescence microscopy, and bright-field microscopy

(a) H\&E stain of a popliteal lymph node with no evidence of metastatic melanoma. (b) Corresponding bright-field image confirming no black melanin pigmentation. (c)

Corresponding fluorescence image of the same lymph node with no GFP expression. (d) H\&E stain of a popliteal lymph node with metastatic melanoma. (e) Corresponding brightfield image confirming the presence of black-pigmented melanoma cells. (f) Fluorescence image of the same lymph node displaying virally mediated green fluorescence in metastatic melanoma cells. 
Table 1

Operationg Characteristics of Virally-Mediated Flourescence Detection of Lymph Node Metastases

\begin{tabular}{ll}
\hline Sensitivity & $80 \%$ \\
Specificity & $100 \%$ \\
PPV & $100 \%$ \\
NPV & $93 \%$ \\
\hline
\end{tabular}

